\title{
Emergency front of neck access after a can't intubate can't oxygenate scenario in a patient with achondroplasia
}

\author{
Adam G. R. Humble, MD (1) - Tom Phu, MD, FRCPC • Kenneth Ryan, MD, FRCPC
}

Received: 1 December 2019/Revised: 16 December 2019/Accepted: 16 December 2019/Published online: 2 January 2020

(C) Canadian Anesthesiologists' Society 2020

To the Editor,

Can't intubate can't oxygenate (CICO) scenarios have a reported incidence of 1:50,000 anesthetics. ${ }^{1}$ We present a case of a 35-yr-old male with achondroplasia who required emergency front of neck access (eFONA) for CICO after anesthetic induction for a semi-elective laminectomy. Written consent for publication was obtained. He was scheduled for a L1-L4 laminectomy to address bilateral leg weakness from spinal stenosis, common in achondroplasia. ${ }^{2}$ He was known to have obstructive sleep apnea (OSA), foramen magnum stenosis, and obesity. Airway exam revealed a Mallampati-II airway, a beard, thick and short neck, moderately limited c-spine mobility, normal thyromental distance, normal mouth opening, and good jaw subluxation.

Awake tracheal intubation with a flexible bronchoscope (FB) was discussed, but it was thought the patient could be bag-mask ventilated and intubated using videolaryngoscopy with a backup plan of supraglottic airway (SGA) insertion through which a FB could be used, if needed, for intubation. After pre-oxygenation and thoracic ramping, anesthesia was induced with fentanyl, propofol, and rocuronium. Bag-mask ventilation with two hands and an oral airway was ineffective. Videolaryngoscopy revealed a grade 3 view with swollen and redundant tissue and attempts to pass a styletted

\section{A. G. R. Humble, MD ( $\square)$}

Department of Anesthesiology Pharmacology and Therapeutics, University of British Columbia, Vancouver, BC, Canada

e-mail: ahumble@ualberta.ca

T. Phu, MD, FRCPC · K. Ryan, MD, FRCPC

Department of Anesthesiology and Perioperative Medicine,

Royal Columbian Hospital, New Westminster, BC, Canada endotracheal tube (ETT) were unsuccessful. As desaturation occurred, help was called for and a SGA (LMA Supreme $^{\mathrm{TM}}$, Teleflex, Athlone, Ireland) was placed, which maintained saturations in the upper 80's. We elected to awaken the patient and sugammadex was administered. Nevertheless, as the neuromuscular blockade reversed and the patient lightened, ventilation became impossible and desaturation occurred. The SGA was exchanged for an intubating SGA (FastTrach ${ }^{\mathrm{TM}}$, Teleflex, Athlone, Ireland) and a FB was placed through the SGA, which revealed no identifiable airway structures and intubation was not attempted. (Figure A). Succinylcholine administration slightly improved ventilation but the oxygen saturation did not recover. Using a \#20 scalpel, a vertical neck incision was made followed by digital palpation and horizontal incision through the cricothyroid membrane (CTM). On the first attempt to pass a bougie, the initial CTM incision could not be found. Success was achieved on the second attempt by leaving the scalpel in the CTM and turning it $90^{\circ}$. A bougie was directed caudally through the CTM and a 6.0 ETT was railroaded over the bougie (Figure B). The patient's saturations recovered, end-tidal carbon dioxide confirmed position, and the hemodynamics remained stable. Further surgery was deferred, the patient recovered in the intensive care without neurologic deficits, and he was decannulated one week after receiving his laminectomy one month later.

The potential for a difficult airway is at the forefront of the numerous anesthetic challenges of achondroplasia. The presence of a short neck, large head, and cervical kyphosis can limit c-spine mobility. ${ }^{3}$ Foramen magnum stenosis is common and can cause spinal cord injury during neck hyperextension. This risk can be mitigated with the use of in-line stabilization and videolaryngoscopy. Subglottic stenosis has also been reported in achondroplasia, 

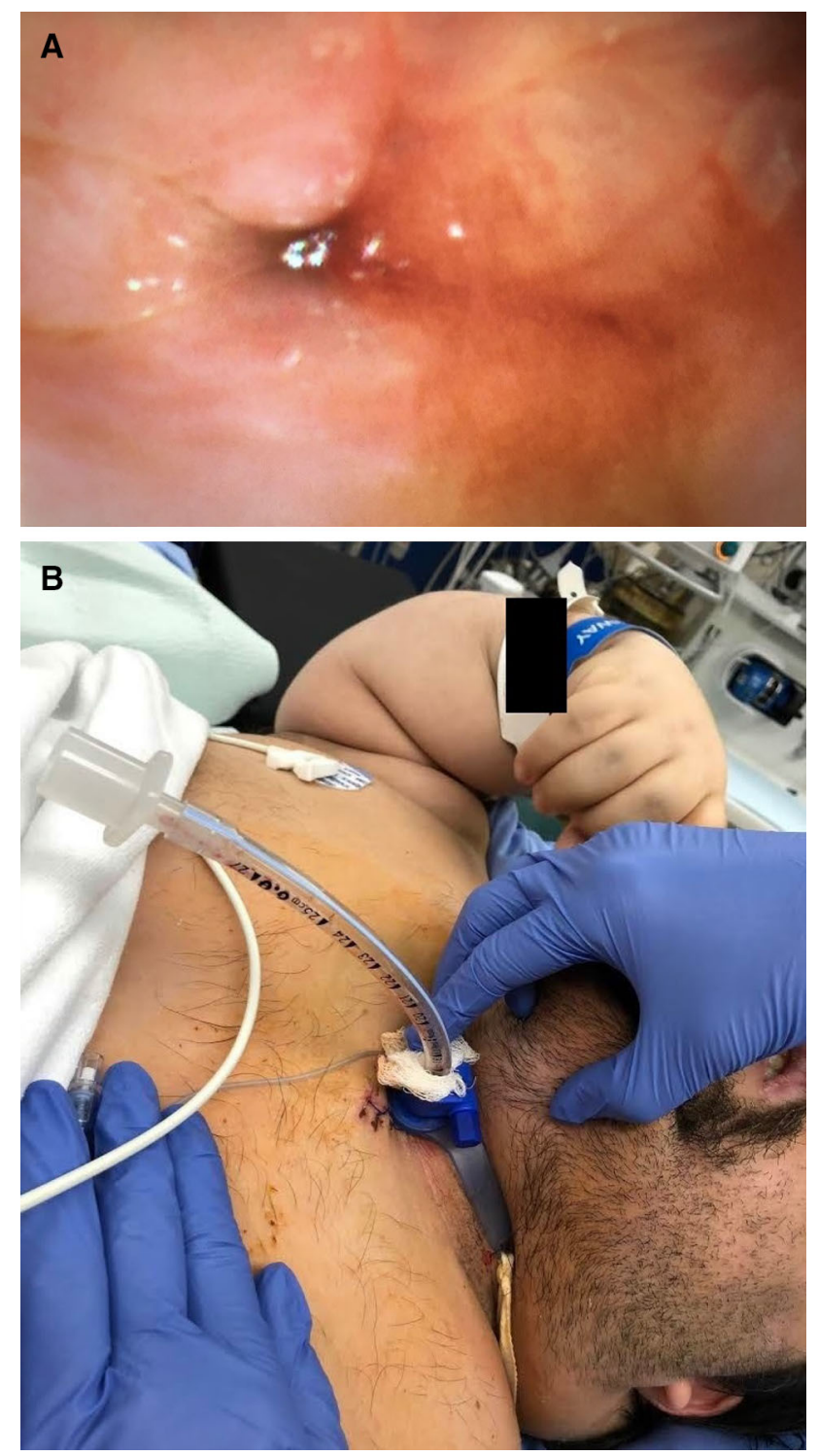

Figure Challenging airway anatomy in a patient with achondroplasia. A) Flexible bronchoscopic view through an intubating supraglottic airway placed after anesthesia induction showing the absence of any identifiable glottic structures. B) An early postoperative image of the cricothyrotomy with a \#6.0 endotracheal tube in situ that allowed for spontaneous ventilation

potentially necessitating the use of smaller ETTs. ${ }^{3}$ Narrow nasal passages can complicate nasal intubations. ${ }^{3}$ Mid-face hypoplasia and adeno-tonsillar hypertrophy contribute to high rates of OSA making bag-mask ventilation potentially difficult. $^{2}$ Restrictive lung physiology complicates ventilation and occurs secondary to hypoplastic ribs, thoracic lordosis, and abdominal obesity. ${ }^{4}$ Pulmonary hypertension can occur as a result of this and should be ruled out in patients with risk factors. ${ }^{3}$ The use of eFONA can be challenging because of the short and thick neck in achondroplasia. $^{4}$

Airway management can be challenging in achondroplasia and a thorough airway plan with support personnel should be in place. We followed the Difficult Airway Society recommendations for a scalpel-bougie approach to eFONA. ${ }^{5}$ All anesthetic carts in our institution are now stocked with a \#10 scalpel, bougie (Pocket Bougie, BOMImed, Bensenville, IL, USA), and a 6.0 ETT.

Funding None

Conflicts of interest None.

Editorial responsibility This submission was handled by Dr. Hilary P. Grocott, Editor-in-Chief, Canadian Journal of Anesthesia.

\section{References}

1. Cook TM, Woodall N, Frerk C; Fourth National Audit Project. Major complications of airway management in the UK: results of the Fourth National Audit Project of the Royal College of Anaesthetists and the Difficult Airway Society. Part 1: Anaesthesia. Br J Anaesth 2011; 106: 617-31.

2. Horton WA, Hall JG, Hecht JT. Achondroplasia. Lancet 2007; 370: $162-72$

3. Huecker M, Harris Z, Yazel E. Occult spinal cord injury after blunt force trauma in a patient with achondroplasia: a case report and review of trauma management strategy. J Emerg Med 2017; 53: 558-62.

4. McCaffer CJ, Douglas C, Wickham MH, Picozzi GL. Acute upper airway obstruction and emergency front of neck access in an achondroplastic patient. BMJ Case Rep 2015; DOI: https://doi.org/ 10.1136/bcr-2015-209614.

5. Frerk C, Mitchell VS, McNarry AF, et al. Difficult Airway Society 2015 guidelines for management of unanticipated difficult intubation in adults. Br J Anaesth 2015; 115: 827-48.

Publisher's Note Springer Nature remains neutral with regard to jurisdictional claims in published maps and institutional affiliations. 